\title{
THE MONTE CARLO COMPLEXITY OF FREDHOLM INTEGRAL EQUATIONS
}

\author{
STEFAN HEINRICH AND PETER MATHÉ
}

\begin{abstract}
A complexity study of Monte Carlo methods for Fredholm integral equations is carried out. We analyze the problem of computing a functional $\mu(y)$, where $y$ is the solution of a Fredholm integral equation

$$
y(s)=\int_{I^{m}} k(s, t) y(t) d t+f(s), \quad s \in I^{m},
$$

on the $m$-dimensional unit cube $I^{m}$, where the kernel $k$ and right-hand side $f$ are given $r$ times differentiable functions. We permit stochastic numerical methods which can make use of function evaluations of $k$ and $f$ only.

All Monte Carlo methods known to the authors for solving the above problem are of the order $n^{-1 / 2}$, while the optimal deterministic methods yield rate $n^{-r /(2 m)}$, thus taking into account the given smoothness of the data. Here, $n$ denotes the (average) number of function evaluations performed. The optimal algorithm we present combines deterministic and stochastic methods in an optimal way. It can be seen that both rates-the standard Monte Carlo rate for general continuous data and the deterministic rate for $r$-smooth data-multiply. This provides the smallest error that stochastic methods of given computational cost can achieve.
\end{abstract}

\section{INTRODUCTION}

The paper is concerned with the efficiency of Monte Carlo methods for the approximate solution of integral equations. We consider Fredholm integral equations of the second kind on the $m$-dimensional unit cube, i.e.,

$$
y(s)=\int_{I^{m}} k(s, t) y(t) d t+f(s), \quad s \in I^{m},
$$

where $I^{m}=[0,1]^{m}$. Here, $k$ and $f$ are given continuous functions on $I^{m} \times I^{m}$ and $I^{m}$, respectively.

Monte Carlo methods are usually applied to find functionals of solutions. To this end, fix a functional $\mu \in C\left(I^{m}\right)^{\prime}$ which is a Radon measure on $I^{m}$. Let us denote the duality between $C\left(I^{m}\right)$ and $C\left(I^{m}\right)^{\prime}$ by $\langle$,$\rangle . The problem we$ study can now be formulated as follows: Given $k$ and $f$ as above, compute $\langle y, \mu\rangle$, where $y$ is the (unique) solution of (1). Of special interest is the case $\mu=\delta_{s_{0}}$, that is, we seek the value of $y$ at $s_{0}, s_{0} \in I^{m}$ fixed. Other cases of interest are $\mu$ being the integral, weighted integral, etc.

Received by the editor November 26, 1990.

1991 Mathematics Subject Classification. Primary 65C05, 65R20. 
We analyze the global behavior of Monte Carlo methods on classes of smooth data, more precisely, on sets of $r$ times continuously differentiable kernels and right-hand sides. We determine the smallest possible error for stochastic methods of given computational cost. To obtain lower bounds, one has to formalize the notion of a Monte Carlo algorithm, which is done in the framework of information-based complexity theory [20]. The upper bounds are established by providing and analyzing a concrete algorithm.

The efficiency of Monte Carlo methods for integration and function approximation was studied in $[3,15,16,20]$. Optimality and complexity of deterministic methods for Fredholm equations, considered over classes of kernels and right-hand sides, were analyzed in $[6,17,18,19,11,12]$. There is also a vast literature on various stochastic methods for Fredholm integral equations (see $[7,9]$ and references therein). However, the rigorous mathematical investigation of the efficiency and complexity aspect of Monte Carlo methods is new, in particular the determination of optimal rates.

Our results, combined with those of Emelyanov and Ilin [6], also allow a certain comparison of deterministic and stochastic methods (which can, of course, only be a rough, heuristic one, owing to the different error criteria used). In this light the algorithm providing the best rate has some new features. The usual Monte Carlo methods yield the typical rate of $M^{-1 / 2}$ for $M$ trials and do not react to any smoothness of the data (see [7]). Deterministic methods, in turn, do so, but yield only a dimension-dependent best rate of $M^{-r /(2 m)}$ ( $M$ the number of arithmetic operations performed; see [6]). Our algorithm keeps both advantages, that is, respects smoothness and adds the Monte Carlo contribution, in this way giving the optimal rate of $M^{-r /(2 m)-1 / 2}$. This is achieved by suitably combining standard deterministic and stochastic methods. Thus, the algorithm we present might also be of computational interest.

The paper is organized as follows. In $\S 2$ we provide the necessary notions and formulate the main results. Proofs of the lower and upper bounds are given in $\S \S 3$ and 4, respectively. Section 5 contains some discussion of models of computation, the computational cost of the concrete algorithm, and the relation to deterministic methods. Basic references for Monte Carlo methods are [7, 9], and for information-based complexity, [20].

\section{THE MAIN RESULT}

First we introduce the classes of right-hand sides and kernels we shall consider. Let $C^{r}\left(I^{m}\right)$ and $C^{r}\left(I^{m} \times I^{m}\right)$ be the spaces of $r$ times continuously differentiable functions (that means-to avoid ambiguity-the restrictions of $C^{r}$-functions defined on some neighborhood of the respective domains).

Let \|\|$_{0}$ denote the maximum norm on $C\left(I^{m}\right)$ and let \|\|$_{r}$ be the norm on $C^{r}\left(I^{m}\right)$ defined by

$$
\|f\|_{r}=\max _{|a| \leq r}\left\|D^{a} f\right\|_{0}
$$

where $a=\left(\alpha_{1}, \ldots, \alpha_{m}\right)$ is a multi-index and $D^{a}$ stands for the respective partial derivative. The corresponding notation applies, of course, to

$$
C^{r}\left(I^{m} \times I^{m}\right)=C^{r}\left(I^{2 m}\right) .
$$


Given constants $\alpha, \beta>0,0<\gamma<1$, and $r \in \mathbf{N}$, we denote

$$
\mathscr{K}^{r}(\alpha, \gamma)=\left\{k \in C^{r}\left(I^{m} \times I^{m}\right),\|k\|_{0} \leq \gamma, \text { and }\|k\|_{r} \leq \alpha\right\}
$$

and

$$
\mathscr{F}^{r}(\beta)=\left\{f \in C^{r}\left(I^{m}\right),\|f\|_{r} \leq \beta\right\} .
$$

The symbol Id always denotes the identity operating in $C\left(I^{m}\right)$. Let $T_{k}: C\left(I^{m}\right)$ $\rightarrow C\left(I^{m}\right)$ denote the integral operator defined for $k \in C\left(I^{m} \times I^{m}\right)$ and $f \in$ $C\left(I^{m}\right)$ by

$$
\left(T_{k} f\right)(s)=\int_{I^{m}} k(s, t) f(t) d t .
$$

The requirement $\|k\|_{0} \leq \gamma$ implies $\left\|T_{k}\right\|_{C\left(I^{m}\right) \rightarrow C\left(I^{m}\right)} \leq \gamma$. From now on we suppose $r, \alpha, \beta, \gamma$ to be fixed. Denote

$$
X=X_{\alpha, \beta, \gamma}^{r}=\mathscr{K}^{r}(\alpha, \gamma) \times \mathscr{F}^{r}(\beta) .
$$

Let finally $\mu \in C\left(I^{m}\right)^{\prime}$ be fixed. Then the solution operator $S_{\mu}: X \rightarrow \mathbf{R}$ is the operator which maps the data $(k, f)$ to the solution $\langle y, \mu\rangle$ of our problem, that is,

$$
S_{\mu}(k, f)=\left\langle\left(\operatorname{Id}-T_{k}\right)^{-1} f, \mu\right\rangle .
$$

Note that the solution operator is nonlinear in $k$.

As a framework in which to study the numerical approximation of $S_{\mu}$ we use the theory of information-based complexity of Traub, Wasilkowski, and Woźniakowski [20].

Let $\mathscr{N}_{\mathrm{st}}^{n}\left(X, \mathbf{R}^{n}\right)$ be the class of all standard information operators from $X$ to $\mathbf{R}^{n}$, that is, the class of mappings of the form

$$
N=\left(\delta_{\left(s_{1}, t_{1}\right)}, \delta_{\left(s_{2}, t_{2}\right)}, \ldots, \delta_{\left(s_{l}, t_{l}\right)}, \delta_{s_{l+1}}, \ldots, \delta_{s_{n}}\right),
$$

i.e.,

$$
N(k, f):=\left(k\left(s_{1}, t_{1}\right), \ldots, k\left(s_{l}, t_{l}\right), f\left(s_{l+1}\right), \ldots, f\left(s_{n}\right)\right),
$$

where $0 \leq l \leq n$ and $s_{i}, t_{j} \in I^{m} \quad(i=1, \ldots, n, j=1, \ldots, l)$. We adopt the convention that $\mathscr{N}_{\text {st }}^{0}\left(X, \mathbf{R}^{0}\right)=\{0\}, 0$ the zero mapping. The quantity $N(k, f)$ represents the information about the data $(k, f)$, which we shall use in the computational process.

Given $N \in \mathcal{N}_{\mathbf{s t}}^{n}\left(X, \mathbf{R}^{n}\right)$, we denote by $\Phi(N, \mathbf{R})$ the class of all mappings $\varphi: N(X) \rightarrow \mathbf{R}$. These mappings are called algorithms using information $N$. The number $\varphi(N(k, f))$ represents the outcome of the computational process performed at the respective information.

The class $\mathscr{M}^{n}(X, \mathbf{R})$ is formed by the composition of some information $N$ and algorithm $\varphi$ :

$$
\mathscr{M}^{n}(X, \mathbf{R})=\left\{\varphi \circ N, \varphi \in \Phi(N, \mathbf{R}), N \in \mathscr{N}_{\mathrm{st}}^{n}\left(X, \mathbf{R}^{n}\right)\right\} .
$$

The elements of $\mathscr{M}^{n}(X, \mathbf{R})$ are called (deterministic) methods-they symbolize the full computational process. Finally,

$$
\mathscr{M}(X, \mathbf{R}):=\bigcup_{n \in \mathbf{N}} \mathscr{M}^{n}(X, \mathbf{R})
$$

constitutes the class of all methods using standard information of some finite cardinality. The elements of $\mathscr{M}(X, \mathbf{R})$ are denoted by $u$. 
Our definition of a random method follows [20, p. 417] (compare also [15]). Define the following $\sigma$-algebra:

$$
\begin{array}{r}
\mathscr{F}(X, \mathbf{R}):=\sigma(\{u \in \mathscr{M}(X, \mathbf{R}), u(k, f) \in A\}, \\
(k, f) \in X \text { and } A \subset \mathbf{R} \text { a Borel set }) .
\end{array}
$$

It follows that for every $(k, f) \in X$ the mapping $u \rightarrow u(k, f)$ is a real random variable on $[\mathscr{M}(X, \mathbf{R}), \mathscr{F}(X, \mathbf{R})]$.

Quite generally, we shall call any probability $P$ on $[\mathscr{M}(X, \mathbf{R}), \mathscr{F}(X, \mathbf{R})]$ a Monte Carlo method. This way we have a large abstract class, containing, in particular, the standard Monte Carlo methods. Furthermore, the cardinality function card: $\mathscr{M}(X, \mathbf{R}) \rightarrow \mathbf{N}$ is defined by

$$
\operatorname{card}(u)=\min \left\{n \in \mathbf{N}, u \in \mathscr{M}^{n}(X, \mathbf{R})\right\} .
$$

This function is possibly not measurable. Therefore, we define the MC-cardinality of a Monte Carlo method $P$ via an upper integral

$$
\begin{aligned}
\operatorname{MC}-\operatorname{card}(P) & =\int_{\mathscr{M}(X, \mathbf{R})}^{*} \operatorname{card}(u) d P(u) \\
& :=\inf \left\{\int_{\mathscr{M}(X, \mathbf{R})} z(u) d P(u), \quad z(u) \geq \operatorname{card}(u)\right.
\end{aligned}
$$

for all $u$ and $z$ is $\mathscr{F}(X, \mathbf{R})$-measurable $\}$.

This quantity plays a crucial role, since it is closely tied to the computational cost of the method (see $\S 5)$. By $\mathscr{P}^{n}(X, \mathbf{R})$ we denote the class of all Monte Carlo methods with MC-cardinality $\leq n$.

The error of a Monte Carlo method $P$ for $S_{\mu}$ at $(k, f) \in X$ is defined by

$$
e^{m c}\left(S_{\mu},(k, f), P\right)=\int_{\mathscr{M}(X, \mathbf{R})}\left|S_{\mu}(k, f)-u(k, f)\right| d P(u),
$$

and the error over the class $X$ by

$$
e^{m c}\left(S_{\mu}, X, P\right)=\sup _{(k, f) \in X} e^{m c}\left(S_{\mu},(k, f), P\right) .
$$

The quantity we are interested in is the smallest error a Monte Carlo method of given cardinality can achieve:

$$
e_{n}^{m c}\left(S_{\mu}, X\right):=\inf \left\{e^{m c}\left(S_{\mu}, X, P\right), P \in \mathscr{P}^{n}(X, \mathbf{R})\right\} .
$$

The main result of the paper is the following.

Theorem 1. For all $r \in \mathbf{N}, \alpha, \beta>0$, and $0<\gamma<1$ there are constants $0<c<C<\infty$ such that we have for $X=X_{\alpha, \beta, \gamma}^{r}$

$$
c n^{-r /(2 m)-1 / 2} \leq \sup _{\|\mu\| \leq 1} e_{n}^{m c}\left(S_{\mu}, X\right) \leq C n^{-r /(2 m)-1 / 2}, \quad n \in \mathbf{N} .
$$

In particular, we get a general lower bound for all Monte Carlo methods using (on the average) not more than $n$ values of the kernel and right-hand side. Since our estimate is sharp up to a constant factor, it also says that there 
is an abstract Monte Carlo method achieving this order of error. One may ask whether there are concrete, practicable algorithms with this property. This is indeed the case. Our proof of the upper bound consists in the description and analysis of such a Monte Carlo method. Theorem 1 also provides the basis of the complexity analysis. Let $\operatorname{MC}-\operatorname{comp}\left(S_{\mu}, X, \varepsilon\right)$ denote the complexity of solving the problem $S_{\mu}$ on $X$ with error at most $\varepsilon$ (see $\S 5$ for a detailed discussion). Then we have

Corollary 1. For all $r \in \mathbf{N}, \alpha, \beta>0$, and $0<\gamma<1$ there are constants $0<c<C<\infty$ and $\varepsilon_{0}>0$ such that we have for $X=X_{\alpha, \beta, \gamma}^{r}$

$$
\begin{aligned}
c\left(\frac{1}{\varepsilon}\right)^{2 m /(r+m)} & \leq \sup _{\|\mu\| \leq 1} \operatorname{MC}-\operatorname{comp}\left(S_{\mu}, X, \varepsilon\right) \\
& \leq C\left(\frac{1}{\varepsilon}\right)^{2 m /(r+m)}, \quad 0<\varepsilon<\varepsilon_{0} .
\end{aligned}
$$

For a comparison, the corresponding deterministic counterpart of Theorem 1 is of interest, which follows from the results of Emelyanov and Ilin [6] (see $\S 5$ for more details). The deterministic rate is $n^{-r /(2 m)}$ (and the corresponding complexity is $\left.\left(\frac{1}{\varepsilon}\right)^{2 m / r}\right)$. So the optimal Monte Carlo rate is indeed the standard Monte Carlo rate $n^{-1 / 2}$, improved by the rate of the optimal deterministic approximation, showing how to combine randomization and approximation in the most efficient way.

\section{THE LOWER BOUND}

The proof of the lower bound in (3) of Theorem 1 is based on a technique developed by Bakhvalov [3].

Let $\nu$ be any discrete probability measure on $X$. Denote by

$$
e_{n}\left(S_{\mu}, X, \nu\right)=\inf \left\{\int_{X}\left|S_{\mu}(k, f)-u(k, f)\right| d \nu(k, f), u \in \mathscr{M}^{n}(X, \mathbf{R})\right\}
$$

the error usually called the average-case error with respect to the measure $\nu$. Since $\nu$ is discrete, no measurability problems occur.

Lemma 1. For each $n \in \mathbf{N}$ we have

$$
e_{n}^{m c}\left(S_{\mu}, X\right) \geq \frac{1}{2} e_{2 n}\left(S_{\mu}, X, \nu\right) .
$$

Proof (compare [15, Lemma 2(ii)]). Let $P$ be any Monte Carlo method with MC-cardinality $\mathbf{M C}-\operatorname{card}(P) \leq n$. Given $0<\varepsilon<1$, let $z: \mathscr{M}(X, \mathbf{R}) \rightarrow \mathbf{R}$ be a measurable function such that $z(u) \geq \operatorname{card}(u), u \in \mathscr{M}(X, \mathbf{R})$, and

$$
\int_{\mathscr{M}(X, \mathbf{R})} z(u) d P(u) \leq(1+\varepsilon) n .
$$

Denote $Z^{2 n}=\{u \in \mathscr{M}(X, \mathbf{R}), z(u) \leq 2 n\}$. Then $Z^{2 n} \subset \mathscr{M}^{2 n}(X, \mathbf{R})$ and $Z^{2 n}$ is measurable. Using Chebyshev's inequality, we can conclude from (5) 
that $P\left(Z^{2 n}\right) \geq(1-\varepsilon) / 2$. Moreover, using Fubini's theorem, we obtain

$$
\begin{aligned}
\sup _{(k, f) \in X} \int_{\mathscr{M}(X, \mathbf{R})}\left|S_{\mu}(k, f)-u(k, f)\right| d P(u) \\
\quad \geq \sup _{(k, f) \in X} \int_{Z^{2 n}}\left|S_{\mu}(k, f)-u(k, f)\right| d P(u) \\
\geq \int_{X} \int_{Z^{2 n}}\left|S_{\mu}(k, f)-u(k, f)\right| d P(u) d \nu(k, f) \\
\quad=\int_{Z^{2 n}} \int_{X}\left|S_{\mu}(k, f)-u(k, f)\right| d \nu(k, f) d P(u) \\
\geq P\left(Z^{2 n}\right) \cdot \inf \left\{\int_{X}\left|S_{\mu}(k, f)-u(k, f)\right| d \nu(k, f), u \in Z^{2 n}\right\} \\
\geq((1-\varepsilon) / 2) \inf \left\{\int_{X}\left|S_{\mu}(k, f)-u(k, f)\right| d \nu(k, f), u \in \mathscr{M}^{2 n}(X, \mathbf{R})\right\} .
\end{aligned}
$$

With $\varepsilon \rightarrow 0$ we arrive at

$$
e_{n}^{m c}\left(S_{\mu}, X\right) \geq \frac{1}{2} e_{2 n}\left(S_{\mu}, X, \nu\right) .
$$

Thus, we have reduced the problem of Monte Carlo methods to an averagecase problem with deterministic methods of fixed cardinality. Moreover, to prove lower bounds, we can specify the functional $\mu$ to be $\sigma=\delta_{s_{0}}$ for some fixed $s_{0} \in I^{m}$.

In fact we shall prove the lower bound for all such $\delta_{s_{0}}$. We can assume without loss of generality that $1 / 2 \leq s_{01} \leq 1$, where $s_{0}=\left(s_{01}, \ldots, s_{0 m}\right)$ (otherwise we modify the subsequent constructions in an obvious way). The following lemma makes it easier to handle the error. Let $\lambda$ denote the integration functional on $C\left(I^{m} \times I^{m}\right)$, that is,

$$
\langle k, \lambda\rangle=\int_{I^{m}} \int_{I^{m}} k(s, t) d s d t, \quad k \in C\left(I^{m} \times I^{m}\right) .
$$

Let $\theta=\frac{1}{2} \min (\alpha, \gamma)$. Then $\theta$, as a constant function, belongs to $\mathscr{K}^{r}(\alpha, \gamma)$, and $T_{\theta} f=\theta \int_{I^{m}} f(t) d t$ is a constant function for every function $f$. Put

$$
I_{1}=\left[0, \frac{1}{2}\right] \times I^{m-1}, \quad J_{1}=\left[\frac{1}{2}, 1\right] \times I^{m-1}
$$

and define

$$
\mathscr{B}=\left\{k \in C^{r}\left(I^{m} \times I^{m}\right),\|k\|_{r} \leq \theta, \operatorname{supp}(k) \subset I_{1} \times J_{1}\right\} .
$$

Observe that for $k \in \mathscr{B}$ we have $\theta+k \in \mathscr{K}^{r}(\alpha, \gamma), T_{k}^{2}=0$, and $k\left(s_{0}, t\right)=0$ for all $t \in I^{m}$.

Lemma 2. Let $\beta, \gamma$, and $\sigma=\delta_{s_{0}}$ be fixed as above. For all $k_{1}, k_{2} \in \mathscr{B}$ we have

$$
\left|S_{\sigma}\left(\theta+k_{1}, \beta\right)-S_{\sigma}\left(\theta+k_{2}, \beta\right)\right| \geq \beta \theta\left|\left\langle k_{1}-k_{2}, \lambda\right\rangle\right| .
$$

Proof. Let $k \in \mathscr{B}$. Since $T_{k}^{2}=0$ and $T_{\theta} T_{k} \beta=\beta \theta\langle k, \lambda\rangle$, we easily verify the equation

$$
\begin{aligned}
\left(\mathrm{Id}-T_{\theta+k}\right)\left(\mathrm{Id}+T_{k}\right) \beta & =\left(\operatorname{Id}-T_{\theta}\left(\mathrm{Id}+T_{k}\right)\right) \beta \\
& =\beta(1-\theta-\theta\langle k, \lambda\rangle) .
\end{aligned}
$$


Since $1-\theta-\theta\langle k, \lambda\rangle$ cannot vanish, we see that

$$
\left(\mathrm{Id}-T_{\theta+k}\right)^{-1} \beta=\frac{1}{1-\theta-\theta\langle k, \lambda\rangle}\left(\mathrm{Id}+T_{k}\right) \beta .
$$

Exploiting, moreover, $k\left(s_{0}, t\right)=0, t \in I^{m}$, we see that

$$
S_{\sigma}(\theta+k, \beta)=\frac{\beta}{1-\theta-\theta\langle k, \lambda\rangle} .
$$

Given kernels $k_{1}, k_{2} \in \mathscr{B}$, we conclude

$$
\begin{aligned}
\mid S_{\sigma}(\theta & \left.+k_{1}, \beta\right)-S_{\sigma}\left(\theta+k_{2}, \beta\right) \mid \\
& =\frac{\beta \theta\left|\left\langle k_{1}-k_{2}, \lambda\right\rangle\right|}{\left(1-\theta-\theta\left\langle k_{1}, \lambda\right\rangle\right)\left(1-\theta-\theta\left\langle k_{2}, \lambda\right\rangle\right)} \geq \beta \theta\left|\left\langle k_{1}-k_{2}, \lambda\right\rangle\right| .
\end{aligned}
$$

Lemma 3. There is a constant $C_{1}>0$ such that for all $n, q \in \mathbf{N}$ with $q \geq 2 n$ and all choices of kernels $k_{1}, \ldots, k_{q} \in \mathscr{B}$ with mutually disjoint supports there is a discrete measure $\nu$ on $X$ such that

$$
e_{2 n}\left(S_{\sigma}, X, \nu\right) \geq C_{1}(q-2 n)^{1 / 2} \min _{1 \leq i \leq q}\left|\left\langle k_{i}, \lambda\right\rangle\right| .
$$

Proof. Let $\left(\varepsilon_{i}\right)_{i=1}^{q}$ be a sequence of independent $\{+1,-1\}$-valued random variables on some probability space $[\Omega, \rho]$ satisfying

$$
\rho\left\{\varepsilon_{i}=-1\right\}=\rho\left\{\varepsilon_{i}=+1\right\}=\frac{1}{2} \text { for all } i
$$

(i.e., they are Bernoulli). Define a mapping $\psi: \Omega \rightarrow X$ by

$$
\psi(\omega)=\left(\theta+\sum_{i=1}^{q} \varepsilon_{i}(\omega) k_{i}, \beta\right) .
$$

That this is indeed a mapping into $X$ follows from the definition of $\theta$ and the assumptions on the $k_{i}$. Let $\nu$ be the image measure $\rho \cdot \psi^{-1}$ induced on $X$ by $\psi$. Now we take any $u \in \mathscr{M}^{2 n}(X, \mathbf{R})$ and represent it as $u=\varphi \cdot N$, $N \in \mathscr{N}_{\mathbf{s t}}^{2 n}\left(X, \mathbf{R}^{2 n}\right), \varphi \in \Phi(N, \mathbf{R})$,

$$
N=\left(\delta_{\left(s_{1}, t_{1}\right)}, \ldots, \delta_{\left(s_{l}, t_{l}\right)}, \delta_{s_{l+1}}, \ldots, \delta_{s_{2 n}}\right) .
$$

Let $A \subset\{1, \ldots, q\}$ be the set of those $i$ for which the support of $k_{i}$ contains at least one of the pairs $\left(s_{1}, t_{1}\right), \ldots,\left(s_{l}, t_{l}\right)$. By assumption, the supports of the $k_{i}$ are disjoint, which implies $\operatorname{card}(A) \leq 2 n$. Let $B=\{1, \ldots, q\} \backslash A$. Then $\operatorname{card}(B) \geq q-2 n$ and

$$
k_{i}\left(s_{j}, t_{j}\right)=0 \quad(i \in B, j=1, \ldots, l) .
$$

Let us denote

$$
\begin{array}{ll}
g_{1}(\omega)=\theta+\sum_{i \in A} \varepsilon_{i}(\omega) k_{i}, & (\omega \in \Omega), \\
g_{2}(\omega)=\sum_{i \in B} \varepsilon_{i}(\omega) k_{i} & (\omega \in \Omega) .
\end{array}
$$

Thus, we have $\psi(\omega)=\left(g_{1}(\omega)+g_{2}(\omega), \beta\right)$ for all $\omega \in \Omega$. Observe that $g_{1}+g_{2}$ and $g_{1}-g_{2}$ have the same distribution and that, by construction,

$$
N\left(g_{1}(\omega)+g_{2}(\omega), \beta\right)=N\left(g_{1}(\omega)-g_{2}(\omega), \beta\right), \quad \omega \in \Omega .
$$


Now we get

$$
\begin{aligned}
& \int_{X}\left|S_{\sigma}(k, f)-u(k, f)\right| d \nu(k, f) \\
& \quad=\int_{\Omega}\left|S_{\sigma}\left(g_{1}(\omega)+g_{2}(\omega), \beta\right)-\varphi\left(N\left(g_{1}(\omega)+g_{2}(\omega), \beta\right)\right)\right| d \rho(\omega) \\
& \quad=\frac{1}{2} \sum_{\tau= \pm 1} \int_{\Omega}\left|S_{\sigma}\left(g_{1}(\omega)+\tau g_{2}(\omega), \beta\right)-\varphi\left(N\left(g_{1}(\omega)+\tau g_{2}(\omega), \beta\right)\right)\right| d \rho(\omega) \\
& \quad \geq \frac{1}{2} \int_{\Omega}\left|S_{\sigma}\left(g_{1}(\omega)+g_{2}(\omega), \beta\right)-S_{\sigma}\left(g_{1}(\omega)-g_{2}(\omega), \beta\right)\right| d \rho(\omega) \\
& \quad \geq \beta \theta \int_{\Omega}\left|\left\langle g_{2}(\omega), \lambda\right\rangle\right| d \rho(\omega)=\beta \theta \int_{\Omega}\left|\sum_{i \in B} \varepsilon_{i}(\omega)\left\langle k_{i}, \lambda\right\rangle\right| d \rho(\omega) \\
& \quad \geq C_{1}\left(\sum_{i \in B}\left\langle k_{i}, \lambda\right\rangle^{2}\right)^{1 / 2} \geq C_{1}(q-2 n)^{1 / 2} \min _{1 \leq i \leq q}\left|\left\langle k_{i}, \lambda\right\rangle\right|,
\end{aligned}
$$

where we used Lemma 2 to derive (6) and Khintchine's inequality with constant $K_{2}$ (see [13, Chapter IV, §5]) to derive (7) with $C_{1}=\beta \theta K_{2}$.

It remains to produce suitable sets of $k_{i}$ 's. Let $\varphi \in C^{\infty}(\mathbf{R}), \operatorname{supp}(\varphi) \subset$ $(0,1)$, and $\int_{0}^{1} \varphi \neq 0$. Let $l \in \mathbf{N}$ and define

$$
\mathscr{J}_{l}=\left\{\left(i_{1}, \ldots, i_{m}\right), 0 \leq i_{1} \leq l-1,0 \leq i_{p} \leq 2 l-1, p=2, \ldots, m\right\},
$$

and

$$
\mathscr{J}_{l}=\left\{\left(j_{1}, \ldots, j_{m}\right), l \leq j_{1} \leq 2 l-1,0 \leq j_{p} \leq 2 l-1, p=2, \ldots, m\right\} .
$$

For $(\mathbf{i}, \mathbf{j}) \in \mathscr{I}_{l} \times \mathscr{J}_{l}, \mathbf{i}=\left(i_{1}, \ldots, i_{m}\right), \mathbf{j}=\left(j_{1}, \ldots, j_{m}\right)$, and $(s, t) \in I^{m} \times I^{m}$, $s=\left(s_{1}, \ldots, s_{m}\right), t=\left(t_{1}, \ldots, t_{m}\right)$, set

$$
\tilde{k}_{(\mathbf{i}, \mathbf{j})}^{l}(s, t):=\prod_{p=1}^{m} \varphi\left(2 l s_{p}-i_{p}\right) \prod_{p=1}^{m} \varphi\left(2 l t_{p}-j_{p}\right) .
$$

It is easily seen that there are constants $C_{2}, C_{3}>0$ (independent of $l$ ) such that for $(\mathbf{i}, \mathbf{j}) \in \mathscr{I}_{l} \times \mathscr{F}_{l}$ we have

$$
\left|\left\langle\tilde{k}_{(\mathbf{i}, \mathbf{j})}^{l}, \lambda\right\rangle\right| \geq C_{2} l^{-2 m}
$$

and

$$
\left\|\tilde{k}_{(\mathbf{i}, \mathbf{j})}^{l}\right\|_{r} \leq C_{3} l^{r}
$$

Finally, let

$$
k_{(\mathbf{i}, \mathbf{j})}^{l}=\theta \frac{\tilde{k}_{(\mathbf{i}, \mathbf{j})}^{l}}{\left\|\tilde{k}_{(\mathbf{i}, \mathbf{j})}^{l}\right\|_{r}} .
$$

Since

$$
\operatorname{supp}\left(k_{(\mathbf{i}, \mathbf{j})}^{l}\right) \subset \prod_{p=1}^{m}\left(\frac{i_{p}}{2 l}, \frac{i_{p}+1}{2 l}\right) \times \prod_{p=1}^{m}\left(\frac{j_{p}}{2 l}, \frac{j_{p}+1}{2 l}\right)
$$


it follows that $k_{(\mathbf{i}, \mathbf{j})}^{l} \in \mathscr{B}$ and that they are of mutually disjoint support. Moreover,

$$
\left|\left\langle k_{(\mathbf{i}, \mathbf{j})}^{l}, \lambda\right\rangle\right| \geq \frac{C_{2}}{C_{3}} l^{-r-2 m}, \quad(\mathbf{i}, \mathbf{j}) \in \mathscr{J}_{l} \times \mathscr{J}_{l} .
$$

Note also that

$$
\operatorname{card}\left(\mathscr{I}_{l} \times \mathscr{J}_{l}\right)=4^{m-1} l^{2 m}
$$

We can accomplish the proof of the lower bound as follows. For each $n \in \mathbf{N}$ let $l_{n}$ be the unique natural number satisfying

$$
4^{m-1} l_{n}^{2 m} \geq 4 n>4^{m-1}\left(l_{n}-1\right)^{2 m} .
$$

Then $l_{n}<\left(2^{(2-m) / m}+1\right) n^{1 /(2 m)}$. Moreover, setting $q_{n}=4^{m-1} l_{n}^{2 m}$, we get $q_{n}-2 n \geq q_{n} / 2$. Now we apply Lemma 3 to $q=q_{n}$ and to the set $\left\{k_{(\mathbf{i}, \mathbf{j})}^{l_{n}}\right.$, $\left.(\mathbf{i}, \mathbf{j}) \in \mathscr{I}_{l_{n}} \times \mathscr{J}_{l_{n}}\right\}$. Combined with Lemma 1 this yields

$$
\begin{aligned}
e_{n}^{m c}\left(S_{\sigma}, X\right) & \geq \frac{C_{1}}{2}\left(\frac{q_{n}}{2}\right)^{1 / 2} \frac{C_{2}}{C_{3}} l_{n}^{-r-2 m} \\
& =2^{m-5 / 2} C_{1} C_{2} C_{3}^{-1} l_{n}^{-r-m} \geq C_{4} n^{-r /(2 m)-1 / 2},
\end{aligned}
$$

which finishes the proof of the lower bound.

\section{THE UPPER BOUND-VARIANCE REDUCTION FOR LIPSCHITZ ESTIMATORS}

Stochastic methods to solve Fredholm equations of the second kind are well known and used frequently (see [7, 9, 8]). All known methods yield the typical Monte Carlo rate $M^{-1 / 2}$ (for $M$ trials), but do not respect the smoothness of the kernel and the right-hand side, respectively.

For the integration problem, among various techniques for variance reduction, the method of "separation of the main part" can be used to include smoothness properties of the integrand (see $[7,8]$ ). This section provides an application of these ideas to Fredholm equations of the second kind, more generally, to numerical problems which allow estimators satisfying some Lipschitz condition.

Precisely, let us introduce the following notion.

Definition 1. Let $(Y, d)$ be a metric space and $\xi=\left(\xi_{x}\right)_{x \in Y}$ be a family of (realvalued) square integrable random variables on a probability space $[\Omega, \mathscr{F}, P]$.

The family $\xi$ is said to be Lipschitz (in mean) if there is a constant $C \geq 0$ such that

$$
\left(\mathbf{E}\left|\xi_{x}-\xi_{y}\right|^{2}\right)^{1 / 2} \leq C d(x, y) .
$$

(The smallest constant $C$ satisfying the above estimate is called the Lipschitz constant.)

Conditions of this type are well known in the theory of stochastic processes and are related to the path continuity (see [14, §35.3]). The following lemma is an immediate consequence of the definition and contains the main idea of the variance-reduction technique.

Lemma 4. Let $(Y, d)$ be a metric space and $\xi=\left(\xi_{x}\right)_{x \in Y}$ be Lipschitz with constant $C$. Then we have

1. $T(x):=\mathbf{E} \xi_{x}, x \in Y$, is Lipschitz with constant $C$ on $Y$. 
2. For $M \in \mathbf{N}$ and independent copies $\xi^{j}$ of $\xi, 1 \leq j \leq M$, define

$$
\zeta_{M}(x):=\frac{1}{M} \sum_{j=1}^{M} \xi_{x}^{j}, \quad x \in Y .
$$

Then we have

$$
\left(\mathbf{E}\left|T(x)-\left[T(y)+\zeta_{M}(x)-\zeta_{M}(y)\right]\right|^{2}\right)^{1 / 2} \leq \frac{C}{\sqrt{M}} d(x, y), \quad x, y \in Y .
$$

Proof. Since $\xi_{x}$ is square integrable, the expectation $\mathbf{E} \xi_{x}$ exists. Moreover, we have

$$
|T(x)-T(y)|=\left|\mathbf{E} \xi_{x}-\mathbf{E} \xi_{y}\right| \leq \mathbf{E}\left|\xi_{x}-\xi_{y}\right| \leq\left(\mathbf{E}\left|\xi_{x}-\xi_{y}\right|^{2}\right)^{1 / 2} \leq C d(x, y) .
$$

To prove (11), we use standard properties of the variance of the sum of independent mean zero randem variables $T(x)-\xi_{x}^{j}$ :

$$
\begin{aligned}
\mathbf{E}\left|T(x)-\left[T(y)+\zeta_{M}(x)-\zeta_{M}(y)\right]\right|^{2} & \\
= & \frac{1}{M^{2}} \mathbf{E}\left|\sum_{j=1}^{M}\right|\left(T(x)-\xi_{x}^{j}\right)-\left(T(y)-\xi_{y}^{j}\right)||^{2} \\
= & \frac{1}{M^{2}} \sum_{j=1}^{M} \mathbf{E}\left|\left(\xi_{y}^{j}-\xi_{x}^{j}\right)-(T(y)-T(x))\right|^{2} \\
& \leq \frac{1}{M} \mathbf{E}\left|\xi_{x}-\xi_{y}\right|^{2} \leq \frac{C^{2}}{M} d^{2}(x, y), \quad x, y \in Y .
\end{aligned}
$$

The Monte Carlo method suggested by the above lemma can be described as follows. Suppose we are given a Monte Carlo method $P$ on $\mathscr{M}(Y, \mathbf{R})$ for a solution operator $S$. This method gives rise to a family $\xi$ of random variables on $[\mathscr{M}(Y, \mathbf{R}), \mathscr{F}(Y, \mathbf{R}), P]$ defined by

$$
\xi_{x}(u):=u(x), \quad x \in Y, u \in \mathscr{M}(Y, \mathbf{R}) .
$$

Further, if $P$ is unbiased for $S$, that means,

$$
S(x)=T(x):=\mathbf{E} \xi_{x}=\int_{\mathscr{M}(Y, \mathbf{R})} u(x) d P(u), \quad x \in Y,
$$

then we can apply the above lemma. Given $x \in Y$, we choose a good approximation $y$ to $x$ for which $T(y)$ can be computed exactly. Knowing $T(y)$, we can improve the approximation by averaging independent trials with Monte Carlo method $P$, each realization of which is applied both to $x$ and $y$. This procedure is working whenever the family $\left(\xi_{x}\right)_{x \in Y}$ of random variables defined by (12) is Lipschitz in mean on an appropriate space $(Y, d)$.

The Monte Carlo methods, applied to solve Fredholm equations of the second kind, are normally based on the use of absorbing Markov chains (see [7, 8, 9]). For our purposes it can be seen that the Markov chain we shall employ has a very simple description. Let $\Delta \in \mathbf{R}^{m} \backslash I^{m}$ be any element, the so-called absorbing state. Let $D:=I^{m} \cup\{\Delta\}$ be the state space, equipped with the Borel $\sigma$-algebra $\mathscr{D}$. Given $0<p<1$ and the (initial) distribution $\mu$ on $I^{m}$, we introduce the distribution of a random sequence $\left(X_{i}\right)_{i=1}^{\infty}$ with values in $[D, \mathscr{D}]$ 
as probability $P$ on $[\Omega, \mathscr{F}]$ with $\Omega=D^{\mathbf{N}}$ and $\mathscr{F}=\mathscr{D}^{\mathbf{N}}$. This is done by giving the transition probabilities. For $n \in \mathbf{N}, x \in I^{m}$, and $\lambda$ the Lebesgue measure on $I^{m}$, let

$$
\begin{gathered}
P\left(X_{1} \in A\right)=\mu(A), \\
P\left(X_{n+1} \in A \mid X_{n}=x\right)=p \cdot \lambda(A), \quad A \subset I^{m}, A \in \mathscr{D}, n \geq 1,
\end{gathered}
$$

and

Further,

$$
P\left(X_{n+1}=\Delta \mid X_{n}=x\right)=1-p .
$$

$$
P\left(X_{n+1}=\Delta \mid X_{n}=\Delta\right)=1, \quad n \geq 1,
$$

meaning that $\Delta$ is absorbing. In the notation of stochastic processes, $\left(X_{i}\right)_{i=1}^{\infty}$ is a stationary Markov chain with absorbing state $\Delta$ and transition densities $p(x, y)=p$. The Markov chain on $[D, \mathscr{D}]$ thus introduced can be completely described by $p$ and the (initial) distribution $\mu$. The lifetime $\tau$ of such a Markov chain $\left(X_{i}\right)_{i=1}^{\infty}$ is defined to be $\tau\left(\left(X_{i}\right)_{i=1}^{\infty}\right):=\min \left\{k, X_{k}=\Delta\right\}-1$ if there is a $k$ with $X_{k}=\Delta$, and $\tau\left(\left(X_{i}\right)_{i=1}^{\infty}\right)=\infty$ otherwise. It will be seen from Proposition 1 below that $\tau$ is finite with probability 1 . The Monte Carlo method will be defined using this Markov chain.

Given $r \in \mathbf{N}, \alpha, \beta, \gamma$, and $X=X_{\alpha, \beta, \gamma}^{r}$ as above, we introduce for technical reasons another space $X_{0}$. To this end, fix $\tilde{\gamma}$ with $\gamma<\tilde{\gamma}<1$ and $\tilde{\beta}$ with $\beta<\tilde{\beta}<\infty$. Put

$$
\begin{array}{r}
X_{0}:=X_{0}(\tilde{\beta}, \tilde{\gamma})=\left\{(k, f), k \in C\left(I^{m} \times I^{m}\right) \text { with }\|k\|_{0} \leq \tilde{\gamma},\right. \\
\left.f \in C\left(I^{m}\right) \text { with }\|f\|_{0} \leq \tilde{\beta}\right\},
\end{array}
$$

equipped with the metric $d((k, f),(h, g)):=\|k-h\|_{0}+\|f-g\|_{0}$. The space $X_{0}$ contains $X$ as a subset, and $S_{\mu}$ can be extended to $X_{0}$ in a natural way, since the defining equation (2) for $S_{\mu}$ also applies to $(k, f) \in X_{0}$. We shall use the same notation $S_{\mu}$ for the extended operator.

First we introduce and analyze the standarci Monte Carlo method, based on this Markov chain (compare [7, 9]). This will be done for elements of $X_{0}$. For this purpose we will specify $p$ and the initial distribution $\mu$. Suppose we are going to approximate $S_{\mu}$ for some $\mu \in C^{\prime}\left(I^{m}\right)$. Since $S_{\mu}$ is linear in $\mu$, and since each $\mu$ can be decomposed into its positive and its negative part, it is easy to see that it is enough to find Monte Carlo methods based on probabilities $\mu$ on $I^{m}$. So, given a probability $\mu_{0}$ on $I^{m}$, we want to approximate $S_{\mu_{0}}$. We choose the Markov chain $\left(X_{i}\right)_{i=1}^{\infty}$ with initial distribution $\mu_{0}$ and transition density $p$ with $\tilde{\gamma}<p<1$, fixed from now on. The distribution of this chain is denoted by $P_{0}$. For each $(k, f) \in X_{0}$ we define a random variable $\xi$ on the probability space $\left[\Omega, \mathscr{F}, P_{0}\right]$ in the following way:

$$
\xi\left(\left(X_{i}\right)_{i=1}^{\infty}, k, f\right):=\left\{\begin{array}{cl}
\frac{1}{(1-p)} f\left(X_{1}\right) & \text { if } \tau\left(\left(X_{i}\right)_{i=1}^{\infty}\right)=1, \\
\frac{1}{(1-p) p^{\tau-1}}\left(\prod_{i=1}^{\tau-1} k\left(X_{i}, X_{i+1}\right)\right) f\left(X_{\tau}\right) \\
\text { if } 1<\tau\left(\left(X_{i}\right)_{i=1}^{\infty}\right)<\infty, \\
\text { otherwise. }
\end{array}\right.
$$

The following proposition contains all technical details needed below. 
Proposition 1. There holds

1. $\mathbf{E} \tau\left(\left(X_{i}\right)_{i=1}^{\infty}\right)=\frac{1}{1-p}$;

2. $\mathbf{E} \xi\left(\left(X_{i}\right)_{i=1}^{\infty}, k, f\right)=S_{\mu_{0}}(k, f),(k, f) \in X_{0}$;

3. The family $\left(\xi_{(k, f)}\right)_{(k, f) \in X_{0}}$ of random variables on $\left[\Omega, \mathscr{F}, P_{0}\right]$, defined by

$$
\xi_{(k, f)}\left(\left(X_{i}\right)_{i=1}^{\infty}\right):=\xi\left(\left(X_{i}\right)_{i=1}^{\infty}, k, f\right), \quad(k, f) \in X_{0},
$$

is Lipschitz with constant

$$
C_{5} \leq \sqrt{\frac{p}{(1-p)\left(p-\tilde{\gamma}^{2}\right)}} \max \left\{1, \frac{\sqrt{2} \tilde{\beta}}{p-\tilde{\gamma}^{2}}\right\} .
$$

Proof. The calculus needed to prove the assertions is standard and explained in [9, Lemma 1.1.1]. Let us recall the following relation, exploited several times below. For convenience, let us introduce the following function:

$$
\chi_{\{n\}}(\tau)= \begin{cases}1, & \tau\left(\left(X_{i}\right)_{i=1}^{\infty}\right)=n \\ 0 & \text { elsewhere }\end{cases}
$$

If $F:\left[D^{\mathbf{N}}, \mathscr{D}^{\mathbf{N}}\right] \rightarrow \mathbf{R}$ is a measurable function and $F_{n}:\left(I^{m}\right)^{n} \rightarrow \mathbf{R}$ defined by $F_{n}\left(x_{1}, \ldots, x_{n}\right)=F\left(x_{1}, \ldots, x_{n}, \Delta, \Delta, \ldots\right)$ is bounded, then we have

$$
\begin{aligned}
\mathbf{E}(F( & \left.\left.\left(X_{i}\right)_{i=1}^{\infty}\right) \cdot \chi_{\{n\}}(\tau)\right) \\
& =\underbrace{\int \cdots \int}_{n \text {-times }} F_{n}\left(x_{1}, \ldots, x_{n}\right) p^{n-1}(1-p) d x_{2} \cdots d x_{n} \mu_{0}\left(d x_{1}\right) .
\end{aligned}
$$

Using (14), we obtain

$$
\mathbf{E} \tau\left(\left(X_{i}\right)_{i=1}^{\infty}\right)=\sum_{n=1}^{\infty} n \mathbf{E} \chi_{\{n\}}(\tau)=\sum_{n=1}^{\infty} n p^{n-1}(1-p)=\frac{1}{1-p},
$$

which proves 1 .

Further,

$$
\begin{aligned}
\mathbf{E} \xi( & \left.\left(X_{i}\right)_{i=1}^{\infty}, k, f\right)=\sum_{n=1}^{\infty} \mathbf{E}\left(\xi\left(\left(X_{i}\right)_{i=1}^{\infty}, k, f\right) \chi_{\{n\}}(\tau)\right) \\
= & \int f\left(x_{1}\right) \mu_{0}\left(d x_{1}\right) \\
& +\sum_{n=2}^{\infty} \underbrace{\int \cdots \int}_{n \text {-times }}\left(\prod_{i=1}^{n-1} k\left(x_{i}, x_{i+1}\right)\right) f\left(x_{n}\right) d x_{n} \cdots d x_{2} \mu_{0}\left(d x_{1}\right) \\
= & \sum_{n=1}^{\infty}\left\langle T_{k}^{n-1} f, \mu_{0}\right\rangle \\
= & \left\langle\left(\operatorname{Id}-T_{k}\right)^{-1} f, \mu_{0}\right\rangle=S_{\mu_{0}}(k, f), \quad(k, f) \in X_{0},
\end{aligned}
$$

proving 2 . 
It remains to prove 3. First observe that $\xi\left(\left(X_{i}\right)_{i=1}^{\infty}, k, f\right)$ is linear in $f$, so that we can conclude

$$
\begin{aligned}
& \xi\left(\left(X_{i}\right)_{i=1}^{\infty}, k, f\right)-\xi\left(\left(X_{i}\right)_{i=1}^{\infty}, h, g\right) \\
& \quad=\xi\left(\left(X_{i}\right)_{i=1}^{\infty}, h, f-g\right)+\xi\left(\left(X_{i}\right)_{i=1}^{\infty}, k, f\right)-\xi\left(\left(X_{i}\right)_{i=1}^{\infty}, h, f\right),
\end{aligned}
$$

and it is sufficient to show that $\xi\left(\left(X_{i}\right)_{i=1}^{\infty}, k, f\right)$ is Lipschitz with constant $C_{5}$ in the second and first arguments, separately. An application of (14) shows

$$
\begin{aligned}
\mathbf{E}\left|\xi\left(\left(X_{i}\right)_{i=1}^{\infty}, h, f-g\right)\right|^{2} & =\frac{1}{1-p} \sum_{n=1}^{\infty}\left\langle T_{h^{2} / p}^{n-1}(f-g)^{2}, \mu_{0}\right\rangle \\
& =\frac{1}{1-p}\left\langle\left(\mathrm{Id}-T_{h^{2} / p}\right)^{-1}(f-g)^{2}, \mu_{0}\right\rangle \\
& \leq \frac{1}{1-p}\left\|\left(\mathrm{Id}-T_{h^{2} / p}\right)^{-1}\right\|_{C\left(I^{m}\right) \rightarrow C\left(I^{m}\right)}\|f-g\|_{0}^{2} .
\end{aligned}
$$

Since $\left\|h^{2} / p\right\|_{0} \leq \tilde{\gamma}^{2} / p$, we obtain

$$
\left(\mathbf{E}\left|\xi\left(\left(X_{i}\right)_{i=1}^{\infty}, h, f-g\right)\right|^{2}\right)^{1 / 2} \leq \sqrt{\frac{p}{\left(p-\tilde{\gamma}^{2}\right)(1-p)}}\|f-g\|_{0} .
$$

The estimate with respect to the kernel is

$$
\begin{aligned}
\mathbf{E} \mid \xi( & \left.\left(X_{i}\right)_{i=1}^{\infty}, k, f\right)-\left.\xi\left(\left(X_{i}\right)_{i=1}^{\infty}, h, f\right)\right|^{2} \\
& =\sum_{n=1}^{\infty} \mathbf{E}\left(\left|\xi\left(\left(X_{i}\right)_{i=1}^{\infty}, k, f\right)-\xi\left(\left(X_{i}\right)_{i=1}^{\infty}, h, f\right)\right|^{2} \cdot \chi_{\{n\}}(\tau)\right) \\
& =\sum_{n=2}^{\infty} \frac{1}{(1-p) p^{n-1}} \underbrace{\int \cdots \int}_{n \text {-times }}\left(\prod_{i=1}^{n-1} k\left(x_{i}, x_{i+1}\right)-\prod_{i=1}^{n-1} h\left(x_{i}, x_{i+1}\right)\right)^{2} \\
& \leq \frac{\|f\|_{0}^{2}}{1-p} \sum_{n=2}^{\infty}\left(\frac{1}{p}\right)^{n-1}(n-1)^{2}\|k-h\|_{0}^{2}\left(\tilde{\gamma}^{2}\right)^{n-2} \\
& \leq \frac{\|f\|_{0}^{2}\|k-h\|_{0}^{2}}{(1-p) \tilde{\gamma}^{2}} \sum_{n=2}^{\infty}(n-1)^{2}\left(\frac{\tilde{\gamma}^{2}}{p}\right)^{n-1} \\
& =\frac{\|f\|_{0}^{2}\|k-h\|_{0}^{2}}{(1-p) \tilde{\gamma}^{2}} \frac{\left(\tilde{\gamma}^{2} / p\right)\left(1+\tilde{\gamma}^{2} / p\right)}{\left(1-\tilde{\gamma}^{2} / p\right)^{3}} \\
& =\frac{\|f\|_{0}^{2}\|k-h\|_{0}^{2}}{(1-p)} \frac{p\left(p+\tilde{\gamma}^{2}\right)}{\left(p-\tilde{\gamma}^{2}\right)^{3}} \leq \frac{2 p x_{2} d \mu_{0}\left(x_{1}\right)}{(1-p)\left(p-\tilde{\gamma}^{2}\right)^{3}}\|k-h\|_{0}^{2} .
\end{aligned}
$$

The derivation of (15) uses the easily verified relation

$$
\left|\prod_{i=1}^{n} a_{i}-\prod_{i=1}^{n} b_{i}\right| \leq n\left(\max _{1 \leq i \leq n}\left(\left|a_{i}\right|,\left|b_{i}\right|\right)\right)^{n-1} \max _{1 \leq i \leq n}\left|a_{i}-b_{i}\right| .
$$


For the value of the sum that gave (16) see [4, 5.2.25]. Hence,

$$
\begin{aligned}
(\mathbf{E} \mid \xi & \left.\left(\left(X_{i}\right)_{i=1}^{\infty}, k, f\right)-\left.\xi\left(\left(X_{i}\right)_{i=1}^{\infty}, h, g\right)\right|^{2}\right)^{1 / 2} \\
\quad \leq & \left(\mathbf{E}\left|\xi\left(\left(X_{i}\right)_{i=1}^{\infty}, k, f\right)-\xi\left(\left(X_{i}\right)_{i=1}^{\infty}, h, f\right)\right|^{2}\right)^{1 / 2} \\
& +\left(\mathbf{E}\left|\xi\left(\left(X_{i}\right)_{i=1}^{\infty}, h, f-g\right)\right|^{2}\right)^{1 / 2} \\
& \leq \sqrt{\frac{2 p \tilde{\beta}^{2}}{(1-p)\left(p-\tilde{\gamma}^{2}\right)^{3}}}\|k-h\|_{0}+\sqrt{\frac{p}{(1-p)\left(p-\tilde{\gamma}^{2}\right)}}\|f-g\|_{0} \\
& \leq \sqrt{\frac{p}{(1-p)\left(p-\tilde{\gamma}^{2}\right)}} \max \left\{1, \frac{\sqrt{2} \tilde{\beta}}{p-\tilde{\gamma}^{2}}\right\}\left(\|k-h\|_{0}+\|f-g\|_{0}\right),
\end{aligned}
$$

which finishes the proof of assertion 3 and of the proposition.

So far, we assembled the facts about the standard Monte Carlo approach. Next we turn to the deterministic setting before we shall combine both to obtain the final, optimal Monte Carlo method. First recall a known fact from approximation theory (see, e.g., [5, Chapters 2.2 and 3.1]).

Proposition 2. There exist constants $\kappa, C_{6}, C_{7}>0$, a function $q: \mathbf{N} \rightarrow \mathbf{N}$ with $q(l) \leq \kappa \cdot l$, a family $\left(\left(t_{i, l}\right)_{i=1}^{q(l)}\right)_{l=1}^{\infty}$ of elements in $I^{m}$, and a family of linearly independent continuous functions $\left(\left(\varphi_{i, l}\right)_{i=1}^{q(l)}\right)_{l=1}^{\infty} \subset C\left(I^{m}\right)$ such that

$$
\begin{gathered}
\sup _{i, l}\left\|\varphi_{i, l}\right\|_{0}<\infty, \\
\max _{i, l} \operatorname{card}\left\{j: \operatorname{supp}\left(\varphi_{i, l}\right) \cap \operatorname{supp}\left(\varphi_{j, l}\right) \neq \varnothing\right\}<\infty .
\end{gathered}
$$

The mappings $P_{l}: C\left(I^{m}\right) \rightarrow C\left(I^{m}\right)$ and $Q_{l}: C\left(I^{m} \times I^{m}\right) \rightarrow C\left(I^{m} \times I^{m}\right)$, defined by

$$
\left(P_{l} f\right)(s):=\sum_{i=1}^{q(l)} f\left(t_{i, l}\right) \varphi_{i, l}(s)
$$

and

$$
\left(Q_{l} k\right)(s, t)=\sum_{i=1}^{q(l)} \sum_{j=1}^{q(l)} k\left(t_{i, l}, t_{j, l}\right) \varphi_{i, l}(s) \varphi_{j, l}(t),
$$

satisfy for $f \in C^{r}\left(I^{m}\right)$ and $k \in C^{r}\left(I^{m} \times I^{m}\right)$

$$
\left\|f-P_{l} f\right\|_{0} \leq C_{6} l^{-r / m}\|f\|_{r}
$$

and

$$
\left\|k-Q_{l} k\right\|_{0} \leq C_{7} l^{-r / m}\|k\|_{r} .
$$

Furthermore, (17) and (18) imply

$$
\sup _{l}\left\|P_{l}\right\|_{C\left(I^{m}\right) \rightarrow C\left(I^{m}\right)}<\infty
$$

and

$$
\sup _{l}\left\|Q_{l}\right\|_{C\left(I^{m} \times I^{m}\right) \rightarrow C\left(I^{m} \times I^{m}\right)}<\infty .
$$

The following fact is well known (see, e,g., [6; 10, Chapter 16]) and expresses the existence of deterministic methods of a certain approximation rate (in fact, the optimal rate for the worst-case setting). 
Proposition 3. There exists a constant $C_{8}>0$ such that for all $l \in \mathbf{N}$ there is an $N_{l} \in \mathcal{N}_{\mathrm{st}}^{l^{2}}\left(X, \mathbf{R}^{l^{2}}\right)$ and a mapping $\psi_{l}: N_{l}(X) \rightarrow C\left(I^{m}\right)$ such that with $v_{l}=\psi_{l} \circ N_{l}$ and $R(k, f):=\left(\operatorname{Id}-T_{k}\right)^{-1} f,(k, f) \in X_{0}$,

$$
\sup _{(k, f) \in X}\left\|v_{l}(k, f)-R(k, f)\right\|_{0} \leq C_{8} l^{-r / m} .
$$

Given $(k, f) \in X$, and using both Proposition 2 and Proposition 3, we can find $(h, g) \in X_{0}$ near $(k, f)$ such that the solution $R(h, g)$ can be given exactly. For this purpose, we define

$$
w_{l}(k, f):=(h, g):=\left(Q_{l} k,\left(\operatorname{Id}-T_{Q_{l}}\right) P_{l} v_{l}(k, f)\right) .
$$

Lemma 5. There exist $C_{9}>0$ and $l_{0} \in \mathbf{N}$ such that for all $l \geq l_{0}$ the transformation $w_{l}$ maps $X$ into $X_{0}$,

$$
\sup _{(k, f) \in X} d\left((k, f), w_{l}(k, f)\right) \leq C_{9} l^{-r / m}, \quad l \geq l_{0},
$$

and

$$
S_{\mu_{0}}\left(w_{l}(k, f)\right)=\left\langle P_{l} v_{l}(k, f), \mu_{0}\right\rangle .
$$

Proof. Let $l_{1}$ be such that for all $k \in \mathscr{K}^{r}(\alpha, \gamma)$

$$
\|k-h\|_{0} \leq C_{7} l^{-r / m}\|k\|_{r} \leq C_{7} \alpha l^{-r / m} \leq \tilde{\gamma}-\gamma, \quad l \geq l_{1} .
$$

This yields $\|h\|_{0} \leq \tilde{\gamma}$ for $l \geq l_{1}$. For $(k, f) \in X$ we have

$$
\begin{aligned}
\| f- & \left(\mathrm{Id}-T_{h}\right) P_{l} v_{l}(k, f) \|_{0} \\
\leq & \left\|\left(\mathrm{Id}-T_{k}\right) R(k, f)-\left(\mathrm{Id}-T_{h}\right) R(k, f)\right\|_{0} \\
& +\left\|\left(\mathrm{Id}-T_{h}\right) R(k, f)-\left(\operatorname{Id}-T_{h}\right) P_{l} R(k, f)\right\|_{0} \\
& +\left\|\left(\operatorname{Id}-T_{h}\right) P_{l} R(k, f)-\left(\operatorname{Id}-T_{h}\right) P_{l} v_{l}(k, f)\right\|_{0} \\
\leq & C_{10} l^{-r / m} .
\end{aligned}
$$

Here we made use of (21), (22), (23), and (24), together with the fact that $\left(\mathrm{Id}-T_{k}\right)^{-1}$ is uniformly bounded on $C^{r}\left(I^{m}\right)$ for $k \in \mathscr{K}^{r}(\alpha, \gamma)$. Now we choose $l_{2}$ in such a way that for $l \geq l_{2}$

$$
\left\|f-\left(\mathrm{Id}-T_{h}\right) P_{l} v_{l}(k, f)\right\|_{0} \leq \tilde{\beta}-\beta,
$$

and put $l_{0}=\max \left\{l_{1}, l_{2}\right\}$. It follows that for $l \geq l_{0}$, the transformation $w_{l}$ maps $X$ into $X_{0}$ and (27) holds. The representation (28) is an immediate consequence of the definitions.

Let us put for $(k, f) \in X_{0}$

$$
\tilde{u}_{l}(k, f)=S_{\mu_{0}}\left(w_{l}(k, f)\right)=\left\langle P_{l} v_{l}(k, f), \mu_{0}\right\rangle .
$$

We are now in a position to describe the Monte Carlo method providing the upper estimate in (3). For each $l \geq l_{0}$ and $M \in \mathbf{N}$ we shall define a Monte Carlo method $P_{l, M}$ on $[\mathscr{M}(X, \mathbf{R}), \mathscr{F}(X, \mathbf{R})]$ as the distribution of a random variable $u_{\omega_{M}}$ on

$$
\left[\left(D^{\mathbf{N}}\right)^{M},\left(\mathscr{D}^{\mathbf{N}}\right)^{M}, \bigotimes_{j=1}^{M} P_{0}\right]
$$


with values $\mathscr{M}(X, \mathbf{R})$. So let

$$
\omega_{M}=\left(\omega^{1}, \ldots, \omega^{M}\right)=\left(\left(X_{i}^{1}\right)_{i=1}^{\infty}, \ldots,\left(X_{i}^{M}\right)_{i=1}^{\infty}\right) \in\left(D^{\mathbf{N}}\right)^{M}
$$

be such that $1 \leq \tau\left(\omega^{j}\right)<\infty$ for $j=1, \ldots, M$ (which holds for almost all $\left.\omega_{M}\right)$. Define for $(k, f) \in X$

$$
u_{\omega_{M}}(k, f)=\tilde{u}_{l}(k, f)+\frac{1}{M} \sum_{j=1}^{M}\left(\xi\left(\omega^{j}, k, f\right)-\xi\left(\omega^{j}, w_{l}(k, f)\right)\right)
$$

The components of $u_{\omega_{M}}$ are defined by (29), Proposition 3, (26), and (13). A look at these relations shows that $u_{\omega_{M}}$ "uses" the following information:

$$
N_{\omega_{M}}=\left(V_{l}, N_{l}, N_{\omega^{1}}, \ldots, N_{\omega^{M}}\right)
$$

Here, $V_{l} \in \mathcal{N}_{\mathbf{s t}}^{q(l)^{2}}\left(X, \mathbf{R}^{q(l)^{2}}\right)$ is defined as

$$
V_{l}(k, f)=\left(k\left(t_{i, l}, t_{j, l}\right)\right)_{i, j=1}^{q(l)}
$$

(used for $Q_{l} k$ in the definition of $\left.w_{l}(k, f)\right)$. The information $N_{l} \in \mathscr{N}_{\mathrm{st}}^{l^{2}}\left(X, \mathbf{R}^{l^{2}}\right.$ ) comes from Proposition 3 (and is used for $\left.v_{l}(k, f)\right)$. For $j=1, \ldots, M$, and $\omega^{j}=\left(X_{i}^{j}\right)_{i=1}^{\infty}$ fixed above, with $1 \leq \tau\left(\omega^{j}\right)<\infty$, the information operator $N_{\omega^{j}} \in \mathscr{N}_{\text {st }}^{\tau\left(\omega^{j}\right)}\left(X, \mathbf{R}^{\tau\left(\omega^{j}\right)}\right)$ is defined as

$$
N_{\omega^{j}}(k, f)= \begin{cases}f\left(X_{1}\right) & \text { if } \tau\left(\omega^{j}\right)=1 \\ \left(\left(k\left(X_{i}, X_{i+1}\right)\right)_{i=1}^{\tau\left(\omega^{j}\right)-1}, f\left(X_{\tau\left(\omega^{j}\right)}\right)\right) & \text { if } \tau\left(\omega^{j}\right)>1\end{cases}
$$

Hence,

$$
N_{\omega_{M}} \in \mathscr{N}_{\mathbf{s t}}^{\eta\left(\omega_{M}, l\right)}\left(X, \mathbf{R}^{\eta\left(\omega_{M}, l\right)}\right),
$$

where $\eta\left(\omega_{M}, l\right)=l^{2}+q(l)^{2}+\sum_{j=1}^{M} \tau\left(\omega^{j}\right)$. In other words, for each $\omega_{M}$ as above, there is a mapping $\varphi_{\omega_{M}} \in \Phi\left(N_{\omega_{M}}, \mathbf{R}\right)$ such that $u_{\omega_{M}}=\varphi_{\omega_{M}} \circ N_{\omega_{M}}$. In order to show that $u_{\omega_{M}}$ is a random variable, we have to verify that the mapping $\omega_{M} \rightarrow u_{\omega_{M}}$ is $\left[\left(D^{\mathbf{N}}\right)^{M}, \mathscr{F}(X, \mathbf{R})\right]$-measurable. But this is easily derived from the structure of $u_{\omega_{M}}$. Consequently, the distribution $P_{l, M}$ of $u_{\omega_{M}}$ is a probability on $[\mathscr{M}(X, \mathbf{R}), \mathscr{F}(X, \mathbf{R})]$, hence a Monte Carlo method. Moreover,

$$
\begin{aligned}
\mathrm{MC}-\operatorname{card}\left(P_{l, M}\right) & \leq \mathbf{E} \eta\left(\omega_{M}, l\right)=l^{2}+q(l)^{2}+\sum_{j=1}^{M} \mathbf{E} \tau\left(\omega^{j}\right) \\
& \leq l^{2}+q(l)^{2}+M \frac{1}{1-p} \\
& \leq C_{11}\left(l^{2}+M\right), \quad l \geq l_{0}, \quad M \in \mathbf{N}
\end{aligned}
$$

Using Proposition 1, we can see that $\mathbf{E} \xi\left(\left(X_{i}\right)_{i=1}^{\infty}, w_{l}(k, f)\right)=S_{\mu_{0}}\left(w_{l}(k, f)\right)=$ $\tilde{u}_{l}(k, f)$ and that the family $\left(\xi_{(k, f)}\right)_{(k, f) \in X_{0}}$ is Lipschitz in mean with constant 
$C_{5}$. Inequalities (11) in Lemma 4 and (27) in Lemma 5 provide the estimate

$$
\begin{aligned}
& \int\left|S_{\mu_{0}}(k, f)-u(k, f)\right| d P_{l, M}(u) \\
& =\int \mid S_{\mu_{0}}(k, f) \\
& \quad-\left\{\tilde{u}(k, f)+\frac{1}{M} \sum_{j=1}^{M}\left(\xi\left(\omega^{j}, k, f\right)-\xi\left(\omega^{j}, w_{l}(k, f)\right)\right)\right\} \mid d P_{0}^{M}\left(\omega_{M}\right) \\
& \leq\left(\int \mid \begin{array}{l}
S_{\mu_{0}}(k, f)-\left\{S_{\mu_{0}}\left(w_{l}(k, f)\right)\right. \\
\leq
\end{array}\right. \\
& \left.\left.\quad+\frac{1}{M} \sum_{j=1}^{M}\left(\xi\left(\omega^{j}, k, f\right)-\xi\left(\omega^{j}, w_{l}(k, f)\right)\right)\right\}\left.\right|^{2} d P_{0}^{M}\left(\omega_{M}\right)\right)^{1 / 2} \\
& \leq C_{5} \cdot \frac{d\left((k, f), w_{l}(k, f)\right)}{\sqrt{M}} \leq C_{5} C_{9} \cdot \frac{l^{-r / m}}{\sqrt{M}}, \quad(k, f) \in X .
\end{aligned}
$$

We conclude

$$
e^{m c}\left(S_{\mu_{0}}, X, P_{l, M}\right) \leq \frac{C_{12}}{\sqrt{M}} l^{-r / m}, \quad l \geq l_{0}, M \in \mathbf{N}
$$

Setting $M=l^{2}$, we conclude

$$
e_{2 C_{11} l^{2}}^{m c}\left(S_{\mu_{0}}, X\right) \leq C_{12} l^{-r / m-1}, \quad l \geq l_{0},
$$

from which the upper estimate in (3) follows. The proof of Theorem 1 is finished.

\section{COMPleXity ANALYSIS}

Besides the order of approximation for a given class of Monte Carlo methods, as expressed by the quantity $e_{n}^{m c}\left(S_{\mu}, X\right)$, another function is of interest, the MC-complexity

$$
\varepsilon \rightarrow \mathrm{MC}-\operatorname{comp}\left(S_{\mu}, X, \varepsilon\right), \quad \varepsilon>0,
$$

describing the average cost of arithmetic operations needed to approximate $S_{\mu}$ with given Monte Carlo error $\leq \varepsilon$. We shall follow the outline in [20, p. 417]. Suppose we are given a function

$$
\theta: \bigcup\left\{\Phi(N, \mathbf{R}), \quad N \in \mathscr{N}_{\mathbf{s t}}^{n}\left(X, \mathbf{R}^{n}\right), n \in \mathbf{N}\right\} \rightarrow \mathbf{R}^{+} \cup\{+\infty\}
$$

(the cost function for the algorithms). Let $c^{*}>0$ be a fixed constant, and define the cost of a deterministic method $u \in \mathscr{M}(X, \mathbf{R})$ by $\operatorname{cost}(u):=\inf \left\{c^{*} n+\theta(\varphi), \varphi \in \Phi(N, \mathbf{R}), N \in \mathscr{N}_{\mathrm{st}}^{n}(X, \mathbf{R}), n \in \mathbf{N}, u=\varphi \circ N\right\}$.

Hence, we assume that we have to pay for the information $N$ (where each component of $N$ is supposed to cost $c^{*}$ ) and for the execution of the algorithm $\varphi$, both independent of $(k, f) \in X$. It follows that $\operatorname{cost}(u) \geq c^{*} \operatorname{card}(u)$. 
We have to make one further assumption. Suppose we can produce a concrete "program" which uses the arithmetic operations $+,-, *, /$ and which computes $\varphi \in \Phi(N, \mathbf{R})$. To be more precise, suppose $\varphi$ can be computed by a straight-line program as defined in [1, 11.2]. Then $\theta(\varphi)$ is assumed to be bounded from above by the number of steps executed by this program. For further details on models of computation and the respective cost functions, we refer to $[1,20]$. To define the cost of a Monte Carlo method, it would be appropriate to average over the cost of the deterministic methods involved. However, we cannot guarantee the $[\mathscr{F}(X, \mathbf{R}), \mathscr{B}(\mathbf{R})]$-measurability. But since cost is positive, we can define the MC-cost of a Monte Carlo method $P$ via an upper integral

$$
\mathrm{MC}-\operatorname{cost}(P):=\int_{\mathscr{M}(X, \mathbf{R})}^{*} \operatorname{cost}(u) d P(u),
$$

as done for the MC-cardinality in $\S 2$. The MC-complexity of the problem $S_{\mu}$ on the class $X$ of problem elements is defined by

$$
\mathrm{MC}-\operatorname{comp}\left(S_{\mu}, X, \varepsilon\right):=\inf \left\{\mathrm{MC}-\operatorname{cost}(P), e^{m c}\left(S_{\mu}, X, P\right) \leq \varepsilon\right\} .
$$

Remark. The given definition of MC-complexity of random methods does not express how hard it is to realize the random process necessary to find $u \in$ $\mathscr{M}(X, \mathbf{R})$. This may be justified if we think of a precomputational process, which provides realizations of all random variables needed to choose $u$. In particular, we assume that we can sample from any given distribution, e.g., $\mu_{0}$, the initial distribution of $P_{0}$ (compare also the remark below).

The above definition (31) of MC-comp $\left(S_{\mu}, X, \varepsilon\right)$, together with the assumptions made on cost, immediately imply

$$
\begin{aligned}
\operatorname{MC}-\operatorname{comp}\left(S_{\mu}, X, \varepsilon\right) & \geq C_{13} \inf \left\{\operatorname{MC}-\operatorname{card}(P), e^{m c}\left(S_{\mu}, X, P\right) \leq \varepsilon\right\} \\
& \geq C_{13} \inf \left\{n-1, e_{n}^{m c}\left(S_{\mu}, X\right) \leq \varepsilon\right\},
\end{aligned}
$$

proving the lower bound in (4).

To prove the upper estimate, we analyze the given algorithm described in $\S 4$. Therefore, we shall briefly outline a verbal description of the stochastic method defined by (30). While the approximative aspects of this method have been handled there, we shall see below that all steps can be executed within the number of operations as claimed in Corollary 1.

First, we have to fix $l \in N$ and put $M:=l^{2}$. Next, points $\left(t_{i, l}\right)_{i=1}^{q(l)}$ in $I^{m}$, a family $\left(\varphi_{i, l}\right)_{i=1}^{q(l)}$ of continuous functions, and mappings $P_{l}, Q_{l}$ from Proposition 2 have to be chosen. Also, $M$ realizations $\omega^{1}, \ldots, \omega^{M}$ of the Markov chain $\left(X_{i}\right)_{i=1}^{\infty}$ can be precomputed, resulting in corresponding sample points.

Now, given $(k, f) \in X$, the following steps have to be performed:

1. Find $v_{l}(k, f)$ according to Proposition 3 in all points $\left(t_{i, l}\right)_{i=1}^{q(l)}$. To do this within the complexity bound, a multilevel method, as, e.g., proposed by Emelyanov and Ilin [6], is required. Thus, we are able to compute $\tilde{u}_{l}(k, f):=$ $\left\langle P_{l} v_{l}(k, f), \mu_{0}\right\rangle$.

2. Compute $\xi\left(\omega^{j}, k, f\right)$ and $\xi\left(\omega^{j}, h, g\right), j=1, \ldots, M$, where $\xi$ is the standard Monte Carlo method defined in (13), and calculate the final approxi- 
mation as

$$
u(k, f)=\tilde{u}_{l}(k, f)+\frac{1}{M} \sum_{j=1}^{M}\left(\xi\left(\omega^{j}, k, f\right)-\xi\left(\omega^{j}, h, g\right)\right)
$$

according to (30). The computation of $\xi\left(\omega^{j}, k, f\right), j=1, \ldots, M$, requires the evaluation of $k$ and $f$ at the sample points. To compute $\xi\left(\omega^{j}, h, g\right), j=$ $1, \ldots, M$, we have to evaluate $h:=Q_{l} k$ and $g:=P_{l} v_{l}(k, f)-T_{h} P_{l} v_{l}(k, f)$ in all points corresponding to $\omega^{1}, \ldots, \omega^{M}$. (Observe that $P_{l} v_{l}(k, f)$ is the exact solution of the Fredholm equation $y(s)=\int_{I^{m}} h(s, t) y(t) d t+g(s), s \in$ $I^{m}$.)

The considerations below will prove that both steps above can be carried out. Step 1 is the deterministic part of the algorithm, while Step 2 forms the stochastic part.

To proceed, we shall count the required arithmetic operations. A look at Step 1 shows that we have to compute $\tilde{u}_{l}(k, f)$. By (29) and (19) we have

$$
\tilde{u}_{l}(k, f)=\left\langle P_{l} v_{l}(k, f), \mu_{0}\right\rangle=\sum_{i=1}^{q(l)} v\left(t_{i, l}\right)\left\langle\varphi_{i, l}, \mu_{0}\right\rangle,
$$

where $v$ stands for $v_{l}(k, f)$. Since $\mu_{0}$ as well as the functions $\varphi_{i, l}$ are known beforehand, the scalar products $\left\langle\varphi_{i, l}, \mu_{0}\right\rangle$ can be precomputed. Moreover, it is known that there are $C_{14}>0$ and $v_{l}$ satisfying Proposition 3 , for which the values $v_{l}(k, f)\left(t_{i, l}\right) \quad(i=1, \ldots, q(l))$ can be computed in $C_{14} l^{2}$ arithmetic operations (see again $[2,6,10])$.

We turn to the randomized part, Step 2. It requires the computation of $M$ realizations of $\xi\left(\left(X_{i}\right)_{i=1}^{\infty}, k, f\right)$ and $\xi\left(\left(X_{i}\right)_{i=1}^{\infty}, w_{l}(k, f)\right)$, respectively. Suppose we are given a realization $\left(x_{i}\right)_{i=1}^{\infty}$ of $\left(X_{i}\right)_{i=1}^{\infty}$ with length $\mathbf{T}=\tau\left(\left(x_{i}\right)_{i=1}^{\infty}\right)$. The representation (13) shows that the calculation of $\xi\left(\left(\ddot{x}_{i}\right)_{i=1}^{\infty}, k, f\right)$ needs no more than $2 \mathrm{~T}$ arithmetic operations. To evaluate $\xi\left(\left(x_{i}\right)_{i=1}^{\infty}, w_{l}(k, f)\right)$, we need to know $h=Q_{l} k$ at $\mathrm{T}-1$ points and $g=P_{l} v_{l}(k, f)-T_{Q_{l} k} P_{l} v_{l}(k, f)$ at another point. The representation (20) shows that the evaluation of $Q_{l} k$ at $\mathbf{T}-1$ points requires the values of $\varphi_{i, l}(s)$ and $\varphi_{j, l}(t)$ at $\mathbf{T}-1$ points. Since any given point $(s, t)$ meets only a constant number of supports (see [18]), we only have to carry out a constant number of evaluations of $\varphi_{i, l}$ at $s$ and $\varphi_{j, l}$ at $t$. In concrete cases, the functions $\varphi_{i, l}(s)$ and $\varphi_{j, l}(t)$ are piecewise polynomials of fixed degree, the pieces and the supports being sets of simple structure (see [5]). So it is clear that the evaluation of $\varphi_{i, l}(s)$ at one point is proportional to the degree, independent of $l$. This implies that the number of arithmetic operations needed to know $Q_{l} k$ at $\mathbf{T}-1$ points requires arithmetic operations proportional to $\mathbf{T}-1$.

The amount of work needed to evaluate $g$ at one point $t$ can be derived from the representation (26) given above,

$$
\begin{aligned}
g(t) & =\sum_{i=1}^{q(l)} v_{l}\left(t_{i, l}\right) \varphi_{i, l}(t)-\sum_{i, j, k=1}^{q(l)} k\left(t_{i, l}, t_{j, l}\right) v_{l}\left(t_{k, l}\right) \varphi_{i, l}(t)\left\langle\varphi_{j, l}, \varphi_{k, l}\right\rangle \\
& =\sum_{i=1}^{q(l)}\left\{v_{l}\left(t_{i, l}\right)-\sum_{j, k=1}^{q(l)} k\left(t_{i, l}, t_{j, l}\right) v_{l}\left(t_{k, l}\right)\left\langle\varphi_{j, l}, \varphi_{k, l}\right\rangle\right\} \varphi_{i, l}(t) .
\end{aligned}
$$


The terms in braces can be computed once for all $t$. Since $\varphi_{i, l}$ are known functions, we can think of $\left\langle\varphi_{j, l}, \varphi_{k, l}\right\rangle$ as being given real numbers. The condition (18) imposed on $\varphi_{i, l}$ shows that the sum over $j, k=1, \ldots, q(l)$, reduces to a sum over a number of summands proportional to $q(l)$. Hence, the computation of the terms in braces for $i=1, \ldots, q(l)$ requires a number of arithmetic operations proportional to $q(l)^{2}$. Once this is done, the computation of $g$ at any given point $t$ requires only a constant number of operations, since the sum over $i=1, \ldots, q(l)$ above reduces to one of constant length. Summarizing the arguments given before, and having the representation (30) in mind, we see that the computation of a realization of $u_{\omega_{M}}(k, f)$ with lengths $\mathbf{T}_{1}, \ldots, \mathbf{T}_{M}$, respectively, requires a number of arithmetic operations bounded by a multiple of $l^{2}+\sum_{i=1}^{M} \mathbf{T}_{i}+q(l)^{2}+M$, which is proportional to $l^{2}+\sum_{i=1}^{M} \mathbf{T}_{i}$. Since we assumed that the algorithmic cost $\theta$ can be estimated in this way, we obtain

$$
\begin{aligned}
\operatorname{MC}-\operatorname{cost}\left(P_{l, M}\right) & =\int^{*} \operatorname{cost}(u) d P_{l, M}(u) \\
& \leq C_{15}\left\{l^{2}+\sum_{j=1}^{M} \int_{\Omega} \tau\left(\omega^{j}\right) d P_{0}^{M}\left(\omega_{M}\right)\right\} \\
& \leq C_{15}\left\{l^{2}+M \mathbf{E} \tau\right\} \leq C_{16}\left(l^{2}+M\right) .
\end{aligned}
$$

The choice of $M=l^{2}$, made to prove the upper estimate in (1), finally yields

$$
\mathrm{MC}-\operatorname{comp}\left(S_{\mu_{0}}, X, \varepsilon\right) \leq C_{17} \cdot(1 / \varepsilon)^{2 m /(r+m)}, \quad \varepsilon>0,
$$

proving Corollary 1.

Remark. Although the model of computation for Monte Carlo methods, introduced above, does not deal with the problem of realizing the random variables needed in the computational process, some hints seem to be useful. A different, but from the point of view of numerical simulation more convenient way to introduce $\left(X_{i}\right)_{i=1}^{\infty}$ is as follows.

Let $\left(Z_{i}\right)_{i=1}^{\infty}$ be a sequence of independent random variables, each with uniform distribution on $[0,1]$. Let $\tau$ be the random natural number, defined as $\tau=\min \left\{i, Z_{i} \geq p\right\}$, i.e.,

$$
P(\tau=n)=p^{n-1}(1-p), \quad n \geq 1 .
$$

Further, let $\left(Y_{i}\right)_{i=2}^{\infty}$ be independent identically distributed with $P_{Y_{i}}=\lambda$, where $\lambda$ is the Lebesgue measure on $I^{m}$. Putting $X_{1}^{\prime}=X_{1}$ distributed according to $\mu_{0}$, and for $i \geq 2$

$$
X_{i}^{\prime}= \begin{cases}Y_{i} & \text { if } i \leq \tau, \\ \Delta & \text { if } i>\tau,\end{cases}
$$

we can easily check that $P_{\left(X_{i}^{\prime}\right)_{i=1}^{\infty}}=P_{\left(X_{i}\right)_{i=1}^{\infty}}$, thus providing the possibility of using a pseudo-random number generator for uniform distributions, readily available on most computers.

It seems worthwhile to make some remarks on the deterministic case of the problem under consideration. Define the error of a deterministic method $u \in$ $\mathscr{M}(X, \mathbf{R})$ by

$$
e\left(S_{\mu}, X, u\right):=\sup \left\{\left|S_{\mu}(k, f)-u(k, f)\right|,(k, f) \in X\right\}
$$


and put

$$
e_{n}\left(S_{\mu}, X\right):=\inf \left\{e\left(S_{\mu}, X, u\right), u \in \mathscr{M}^{n}(X, \mathbf{R})\right\}
$$

Then

$$
c n^{-r /(2 m)} \leq \sup _{\|\mu\| \leq 1} e_{n}\left(S_{\mu}, X\right) \leq C n^{-r /(2 m)} .
$$

This was proved by Emelyanov and Ilin [6]. To draw the final conclusion, we see that for the optimal Monte Carlo method, both rates multiply-the standard Monte Carlo rate for general continuous data, $n^{-1 / 2}$, and the deterministic rate for $r$-smooth data, $n^{-r /(2 m)}$. Statements analogous to (32), and the conclusions above, hold also for the complexity.

\section{BIBLIOGRAPHY}

1. A. V. Aho, J. E. Hopcroft, and J. D. Ullmann, The design and analysis of computer algorithms, Addison-Wesley, Reading, MA, 1974.

2. K. E. Atkinson, A survey of numerical methods for the solution of Fredholm integral equations of the second kind, SIAM, Philadelphia, 1976.

3. N. S. Bakhvalov, On approximate computation of multiple integrals, Vestnik Moskov. Univ. Ser. I Mat. Mekh. 4 (1959), 3-18. (Russian)

4. Yu. A. Brychkov, O. I. Marichev, and A. P. Prudnikov, Integrals and series, Volume 1: Elementary functions, Gordon \& Breach, New York, London, and Paris, 1986.

5. P. G. Ciarlet, The finite element method for elliptic problems, North-Holland, Amsterdam, New York, and Oxford, 1978.

6. K. V. Emelyanov and A. M. Ilin, On the number of arithmetic operations, necessary for the approximate solution of Fredholm integral equations of the second kind, $\mathrm{Zh}$. Vychisl. Mat. i Mat. Fiz. 7 (1967), 905-910; English transl. in USSR Comput. Math. and Math. Phys. 7 (1967), 259-267.

7. S. M. Ermakov, The Monte Carlo method and related problems, Nauka, Moscow, 1971. (Russian)

8. S. M. Ermakov and G. A. Michailov, The theory of statistical trials, 2nd ed., Nauka, Moscow, 1982. (Russian)

9. S. M. Ermakov, W. W. Nekrutkin, and A. S. Sipin, Random processes for classical equations of mathematical physics, Math. Appl. (Soviet Ser.), vol. 34, Kluwer, Dordrecht, 1989.

10. W. Hackbusch, Multi-grid methods and applications, Springer, Berlin, Heidelberg, and New York, 1985.

11. S. Heinrich, On the optimal error of degenerate kernel methods, J. Integral Equations 9 (1985), 251-266.

12. _ـ Probabilistic analysis of numerical methods for integral equations, J. Integral Equations Appl. 3 (1991), 289-319.

13. St. Kaczmarz and H. Steinhaus, Theory of orthogonal series, 2nd ed., Chelsea, New York, 1951.

14. M. Loève, Probability theory, 4th ed., Graduate Texts in Math., vol. 46, Springer, New York, 1978.

15. P. Mathé, Random approximation of Sobolev embeddings, J. Complexity 7 (1991), 261-281.

16. E. Novak, Deterministic and stochastic error bounds in numerical analysis, Springer, Berlin, Heidelberg, and New York, 1988.

17. S. V. Pereverzev, On the optimization of adaptive methods for the approximate solution of integral equations, Dokl. Akad. Nauk SSSR 267 (1982), 1304-1308; English transl. in Soviet Math. Dokl. 26 (1982), 770-774.

18. _ On the complexity of the problem of finding solutions of Fredholm equations of the second kind with smooth kernels. I, Ukrain. Mat. Zh. 40 (1988), 84-91; English transl. in Ukrainian Math. J. 40 (1988), 71-76. 
19. __ On the complexity of the problem of finding solutions of Fredholm equations of the second kind with smooth kernels. II, Ukrain. Mat. Zh. 41 (1989), 189-193; English transl. in Ukrainian Math. J. 41 (1989), 169-173.

20. J. F. Traub, G. W. Wasilkowski, and H. Woźniakowski, Information-based complexity, Academic Press, New York, 1988.

Institut für ANGewandte Analysis und Stochastik, Mohrenstrasse 39, PF 1304, D-O1086 BERLIN, GERMANY 